\title{
Influence of Plasmonic Array Geometry on Non-radiative Energy Transfer from a Quantum Well to a Quantum Dot Layer
}

\author{
Luke J. Higgins, Cristian A. Marocico, Jorge Garcia Coindreau, Vasilios D. Karanikolas, Alan P. Bell, \\ John J. Gough, Graham P. Murphy, Peter J. Parbrook*, and A. Louise Bradley \\ School of Physics and CRANN, Trinity College Dublin, College Green, Dublin 2, Ireland \\ * Tyndall national Institute and School of Engineering, University College Cork, \\ Lee Maltings, Prospect Row, Cork, Ireland \\ Tel: (3531) 8963595,Fax: (3531)8962711,e-mail: bradlel@tcd.ie
}

\begin{abstract}
The influence of ordered plasmonic arrays on energy transfer from a quantum well to a quantum dot layer has been investigated. The ordered arrays are comprised of nanostructures of different geometries, including boxes, disks and rings. Despite no signature of non-radiative energy transfer in the absence of an array, an efficiency of $\sim 51 \%$ is observed for a ring array, though strong emission quenching yields an overall increase of only $\sim 14 \%$ of the QD emission. The QD emission is enhanced by $\sim 25 \%$ for disk arrays, and was found to be relatively insensitive to the gap between disks. In contrast, the QD emission enhancement decreases from $\sim 70 \%$ to $40 \%$ as the separation between boxes increases from $100 \mathrm{~nm}$ to $160 \mathrm{~nm}$. The largest increase in QD emission of $\sim 70 \%$ is due to a non-radiative energy transfer efficiency of $\sim 25 \%$ coupled with a QD emission enhancement factor of 1.4. The results demonstrate the flexibility offered by plasmonic arrays to optimise non-radiative energy transfer or to benefit from a combination of energy transfer and enhanced radiative emission, relevant to sensing and colour conversion applications.
\end{abstract}

Keywords: plasmonics, non-radiative energy transfer, quantum dots, Förster resonance energy transfer (FRET), down conversion, quantum well.

\section{INTRODUCTION}

The control of relaxation pathways in optical emitters using localised surface plasmons (LSPs) supported by noble metal nanostructures has been an intense area of investigation over the past decade. First reports probed examples of emission enhancement and emission quenching through modification of radiative and non-radiative rates, respectively [1,2]. Energy can be transferred to the metal nanoparticles and, subsequently, dissipated as Joules heating. It was predicted that LSPs could enhance the non-radiative energy transfer between a dipole donor and dipole acceptor $[3,4]$. This non-radiative energy transfer, also known as Förster resonance energy transfer (FRET), arises due to the Coulomb interaction between the two dipoles. For a single donor-acceptor dipole pair it exhibits a $d^{-6}$ separation dependence. FRET has been considered for applications in nanosensors, detectors, photovoltaics, down conversion based light emitting devices. However, the strong distance dependence has limited performances[5]. Recent work demonstrating experimentally plasmon enhanced nonradiative energy transfer between donor and acceptor colloidal quantum dots using colloidal Au nanoparticles prompted further investigation of the potential for non-radiative energy transfer in other material systems such as hybrid quantum well (QW)-quantum dot (QD) structures, as used in light harvesting and light emitting diode devices [6,7]. Typically down-conversion light emitting diodes are based on radiative energy transfer from the quantum well to the quantum dots. However, an efficient non-radiative energy transfer process could offer advantages in terms of light extraction from the quantum well and absorption in the QD layer. In this paper a study of the influence of ordered plasmonic arrays, comprised of various individual nanoparticle geometries, on the efficiency of the plasmon enhanced non-radiative energy transfer efficiency and the enhancement of the emission from the QD acceptor layer is presented. Lithographically defined arrays provide flexibility in the selection of the nanostructure shape, uniformity across the surface and a well-controlled range of parameters, such as the dimension of the nanostructure and the pitch of the array. The array properties can influence the absorption, emission and energy transfer processes allowing for tailoring of structures specifically for optimised energy transfer or acceptor emission, as can be exploited for light emitting devices or sensing applications.

\section{EXPERIMENTAL METHODS}

A schematic of the plasmon-mediated QW-QD energy transfer structure is shown in Fig. 1(a). A single $2 \mathrm{~nm}$ thick InGaN QW was grown by metal-organic vapour phase epitaxy on a thick GaN layer on a c-plane sapphire substrate. The QW was capped by a $3 \mathrm{~nm} \mathrm{GaN}$ barrier. The Ag nanostructure arrays were fabricated on the top surface of the GaN barrier using a He-ion lithography technique. A $5 \mathrm{~nm}$ Ti layer was firstly deposited, followed by $35 \mathrm{~nm}$ of $\mathrm{Ag}$, yielding nanostructures with a height of $40 \mathrm{~nm}$. Arrays of boxes, disks and rings of various structure dimensions and array pitch were investigated, with details provided in Table 1. Each array contained 200 x 200 units. All box and disk arrays have a nanostructure of the same dimension, namely a $100 \mathrm{~nm}$ edge 


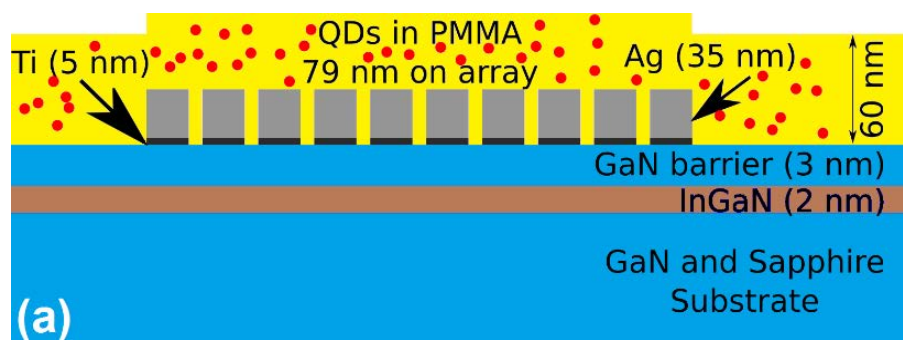

(b)
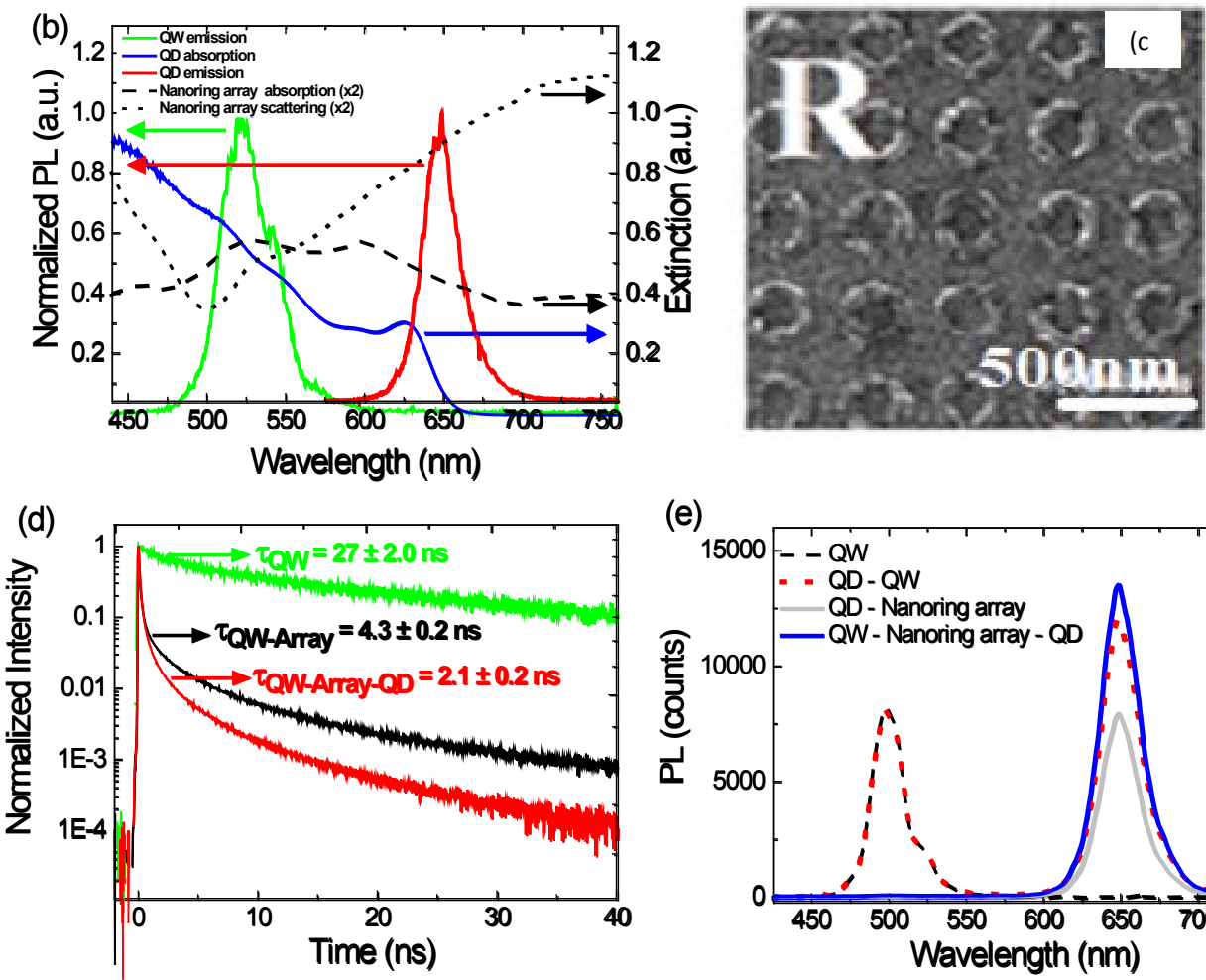

(e)

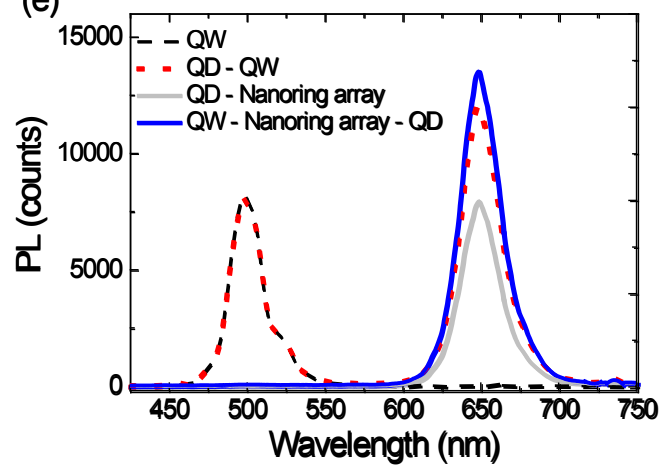

Figure 1: (a) Schematic of the plasmon-enhanced energy transfer structure; (b) Normalised PL spectra of the $Q W$ and $Q D$ layer, scattering and absorption spectra of the ring array and the extinction spectrum of the $Q D$ layer; (c) He-ion micrograph of the ring array; (d) The time-resolved PL decay curves for the $Q W\left(\tau_{O W}\right)$, ring array decorated $Q W\left(\tau_{O W-\text { Array }}\right)$ and full energy transfer structure $\left(\tau_{O W-\text { Array-OD }}\right)$. The PL spectra for the $Q W, Q D$ coated $Q W(Q D-Q W), Q D$ coated array on GaN substrate (QD-Nanoring array) and full energy transfer structure ( $Q W$-Nanoring array- $Q D)$.

length for the boxes and $70 \mathrm{~nm}$ diameter for the disks. The properties of arrays are different to those of the individual nanostructure and by varying the gap between the individual nanostructures the absorption and scattering properties of the arrays can be tuned. The numerically calculated scattering and absorption spectra for the ring array are shown in Fig. 1(b). A He-ion microscopy image is presented in Fig. 1(c). The energy transfer structure is completed by spin coating $6.3 \mathrm{~nm}$ diameter CdSe/ZnS core-shell QDs dispersed in an $80 \mathrm{~nm}$ thick PMMA layer of on top of the Ag nanostructure arrays. The Ag nanostructure arrays were also fabricated on top of bulk GaN for reference measurements.

Spectral and time-resolved photoluminescence (PL) measurements are used to characterise the impact of the Ag nanostructure arrays on the QW emission, QD emission and non-radiative energy transfer process. The PL spectra for the QW and QD layer, as well as the absorption of the QD layer are shown in Fig. 1(b). The donor QW emission peak at $546 \mathrm{~nm}$ is seen to overlap well with the QD absorption spectrum, a requisite condition for FRET. The emission from the QDs in the PMMA layer shows a peak at $649 \mathrm{~nm}$. The PL spectra for the QW, the QD coated QW, the QD coated ring array on the GaN substrate and the full energy transfer structure are shown in Fig. 1(e). Increased QD emission can be seen for the energy transfer structure. The average PL decay lifetimes, shown in Fig. 1(d) for the ring array, can be used to calculate the energy transfer efficiency,

$$
E_{F R E T}=1-\frac{\tau_{Q W-\text { Array }-Q D}}{\tau_{Q W-\text { Array }}}
$$


where $\tau_{Q W-A r r a y}$ and $\tau_{Q W-A r r a y-Q D}$ are the average QW PL decay times in the absence and presence of the QDs, respectively. The QD emission enhancement for the full energy transfer structure is given by

$$
\frac{I_{Q D-\text { Array }-Q W}}{I_{Q D}}-1,
$$

while the QD emission ratio on the GaN substrate is described by

$$
\frac{I_{Q D-\text { Array }}}{I_{Q D}}
$$

where $I_{Q D-A r r a y-Q W}$ is the integrated QD emission on the array on the QW substrate, $I_{Q D}$ is the QD emission off the array and $I_{Q D-A r r a y}$ is the integrated QD emission on the array on the GaN substrate.

\begin{tabular}{|c|c|c|c|}
\hline Geometry & Label & Dimension (nm) & Gap in the array (nm) \\
\hline $\begin{array}{l}\text { Ring } \\
\text { - Inner diameter } \\
\text { - Outer diameter }\end{array}$ & $R$ & $\begin{array}{l}150 \\
250\end{array}$ & 100 \\
\hline $\begin{array}{l}\text { Box } \\
\quad-\text { Edge length }\end{array}$ & $B$ & 100 & $100(\mathrm{~B} 1), 130(\mathrm{~B} 2), 160(\mathrm{~B} 3)$ \\
\hline $\begin{array}{l}\text { Disk } \\
\quad-\text { Diameter }\end{array}$ & $\mathrm{D}$ & 70 & $100(\mathrm{D} 1), 130(\mathrm{D} 2), 160(\mathrm{D} 3)$ \\
\hline
\end{tabular}

Table 1. Nanostructure geometries and dimensions. All structures have a height of $40 \mathrm{~nm}$.

\section{RESULTS AND DISCUSSION}

Measurements of the QW emission in the absence of any array showed no change in the QW lifetime or spectra, thereby confirming that there are no signature of non-radiative energy transfer from the QW to the QDs in the absence of a plasmonic component. Figure 2(a) shows the energy transfer efficiency calculated using Equation (1) for the range of Ag nanostructure arrays. The non-radiative energy transfer is enhanced in all cases. The ring array shows the highest energy transfer efficiency of $\sim 51 \%$. The energy transfer efficiency for the boxes and disks is seen to be significantly lower, varying between $15 \%$ and $30 \%$. It can also be noted that the non-radiative energy transfer efficiency for the box arrays does not show a clear dependence on the gap size across the arrays, while the disk arrays exhibit a decrease in energy transfer efficiency with increasing gap between the disks.
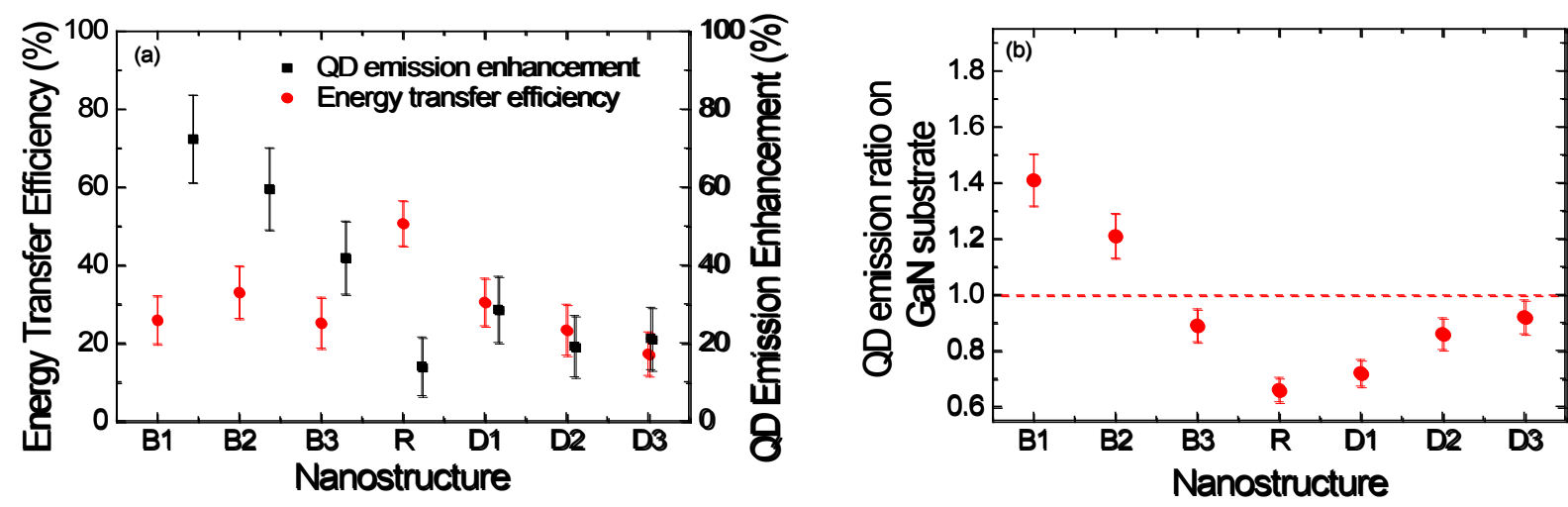

Figure 2: (a) Energy transfer efficiency and QD emission enhancement as a function of array; (b) The ratio of the QD emission measured on and off the array on a GaN substrate as a function of array.

It interesting to compare the array dependence of the non-radiative energy transfer efficiency with that of the QD emission enhancement. The QD emission enhancement is calculated using equation (2) and is also shown in Fig. 2(a). The first thing that can be noted is that all structures show enhancement of the QD emission. Secondly, this enhancement is lowest for the ring array, at just $\sim 14 \%$, in stark contrast to the large energy transfer efficiency. The QD emission is enhanced by $\sim 25 \%$ for the disks, and is relatively insensitive to the gap dimension. The QD emission enhancement is largest for the three box arrays and exhibits a strong dependence on gap, decreasing from $\sim 70 \%$ to $40 \%$ as the gap increases from $100 \mathrm{~nm}$ to $160 \mathrm{~nm}$. These results show that there is significant opportunity to enhance the non-radiative energy transfer efficiency and to increase the 
acceptor emission through the tailoring of nanostructure arrays. The results also clearly show that the influence of the array on the non-radiative energy transfer alone is not a good indicator of the final QD emission. This is expected as it is well-known that plasmonic arrays directly modify emission, causing enhancement or quenching. Experimentally this factor is evaluated by measuring the QD emission on and off the array on the GaN substrate reference samples using equation (3). This QD emission ratio is plotted in Fig. 2(b). Values greater than one correspond enhancement of the QD emission due to the interaction with the plasmonic array, whereas values less than 1 corresponds to emission quenching. The first comment is that only two of the seven arrays show enhancement of the QD emission. The ring array shows the largest quenching, with the QD emission reduced by $40 \%$. The box arrays shows a strong dependence on the gap dimension, in contrast to the relative insensitivity of the disks arrays. The largest increase in the QD emission by $40 \%$ is observed for the box array with the smallest gap of $100 \mathrm{~nm}$. The largest increase in QD emission of $\sim 71 \%$ is due to a non-radiative energy transfer efficiency of $\sim 25 \%$ coupled with an array QD emission ratio of $\sim 1.4$. A model has been developed to confirm that the increases in the QD emission on the array-decorated QW can be fully attributed to plasmon-enhanced nonradiative energy transfer [8].

\section{CONCLUSIONS}

The influence of the geometry of plasmonic arrays on non-radiative energy transfer from an InGaN/GaN QW to an $80 \mathrm{~nm}$ thick layer of CdSe/ZnS QDs dispersed in PMMA was investigated. It has been demonstrated that large increases in the energy transfer efficiency can be achieved. The QD emission can also be increased but does not show the same dependence on the array geometry. There are two notable examples. Firstly, while the highest energy transfer efficiency is observed for a ring array, this yields the lowest overall QD emission. Secondly, while the energy transfer efficiency is relatively insensitive to the gap between the boxes within the array, the QD emission is very sensitive to this array parameter. The results demonstrate the flexibility offered by plasmonic arrays, even for a single nanostructure geometry, to optimise non-radiative energy transfer or to benefit from a combination of energy transfer and enhanced radiative emission, relevant to sensing and colour conversion applications.

\section{ACKNOWLEDGEMENTS}

This work was supported by Science Foundation Ireland (SFI) under grant number 10/IN.1/12975 and the National Access Programme Grant under grant number NAP 338, and enabled using facilities funded by Irish Higher Education Authority Programme for Research in Third Level Institutions Cycles 4 and 5 via the INSPIRE and TYFFANI projects. We also acknowledge the Advanced Microscopy Laboratory (AML) for use of the helium-ion microscope. JJG acknowledges a postgraduate research scholarship from the Irish Research Council (GOIPG/2013/680) and PJP a SFI Engineering Professorship (SFI/07/ EN/E001A).

\section{REFERENCES}

[1] P. Anger, P. Bharadwaj, and L. Novotny, "Enhancement and quenching of single-molecule fluorescence," Phys. Rev. Lett., vol. 96, no. 11, pp. 3-6, 2006.

[2] P. P. Pompa et al., "Metal-enhanced fluorescence of colloidal nanocrystals with nanoscale control.," Nat. Nanotechnol., vol. 1, no. 2, pp. 126-130, 2006.

[3] J. I. Gersten, "Accelerated energy transfer between molecules near a solid particle," Chem. Phys. Lett., vol. 104, no. 1, pp. 31-37, 1984.

[4] A. O. Govorov, J. Lee, and N. A. Kotov, "Theory of plasmon-enhanced Förster energy transfer in optically excited semiconductor and metal nanoparticles," Phys. Rev. B - Condens. Matter Mater. Phys., vol. 76, no. 12 , pp. 1-16, 2007.

[5] M. Achermann et al., "Nanocrystal-based light-emitting diodes utilizing high-efficiency nonradiative energy transfer for color conversion," Nano Lett., vol. 6, no. 7, pp. 1396-1400, 2006.

[6] V. K. Komarala et al., "Surface plasmon enhanced Förster resonance energy transfer between the CdTe quantum dots," Appl. Phys. Lett., vol. 93, no. 12, pp. 2006-2009, 2008.

[7] G. P. Murphy et al., "Ag colloids and arrays for plasmonic non-radiative energy transfer from quantum dots to a quantum well," Nanotechnology, vol. 28, p. 115401, 2017.

[8] L. Higgins et al., "Influence of plasmonic array geometry on energy transfer from a quantum well to a quantum dot layer," Nanoscale, pp. 41-43, 2016. 